Bull. Mater. Sci., Vol. 39, No. 6, October 2016, pp. 1441-1451. (c) Indian Academy of Sciences.

\title{
Bandgap engineered graphene and hexagonal boron nitride for resonant tunnelling diode
}

\author{
PENCHALAIAH PALLA*, GOPI RAJA UPPU, ANITA S ETHIRAJ and J P RAINA \\ Center for Nanotechnology Research, VIT University, Vellore 632014, India
}

MS received 15 September 2015; accepted 21 March 2016

\begin{abstract}
In this article a double-barrier resonant tunnelling diode (DBRTD) has been modelled by taking advantage of single-layer hexagonal lattice of graphene and hexagonal boron nitride (h-BN). The DBRTD performance and operation are explored by means of a self-consistent solution inside the non-equilibrium Green's function formalism on an effective mass-Hamiltonian. Both p- and n-type DBRTDs exhibit a negative differential resistance effect, which entails the resonant tunnelling through the hole and electron bound states in the graphene quantum well, respectively. The peak-to-valley ratio of approximately 8 (3) for p-type (n-type) DBRTD with quantum well of $5.1 \mathrm{~nm}(4.3 \mathrm{~nm})$ at a barrier width of $1.3 \mathrm{~nm}$ was achieved for zero bandgap graphene at room temperature.
\end{abstract}

Keywords. Device simulation; graphene; h-BN; NEGF; RTD.

\section{Introduction}

Low-dimensional nanomaterials, such as monolayer graphene and hexagonal boron nitride (h-BN), have the same twodimensional (2D) honeycomb lattice but drastically different electronic properties: graphene is a gapless semi-metal, while $\mathrm{h}-\mathrm{BN}$ is a semiconductor with a large bandgap (insulator) [1]. Moreover, h-BN has an atomically smooth surface with lattice constant just $1.8 \%$ greater than graphene. Graphene supported on insulating substrates such as $\mathrm{SiC}, \mathrm{SiO}_{2}$ and high- $\kappa$ insulators [2] has reduced electron or hole mobility, which is generally attributed to surface optical phonons, surface roughness and charge surface states. On the other hand, graphene placed on h-BN substrate has shown higher mobility up to $1.25 \times 10^{5} \mathrm{~cm}^{2} \mathrm{Vs}^{-1}$ at room temperature [3], which is more than on any other substrate reported so far [4-6]. This higher mobility is attributed to the fact that the surface of h-BN is free from dangling bonds, atomically flat, relatively chemically inert, with a low density of charged impurities [4]. Because of the above-stated benefits, h-BN has become a very good material to attain the ballistic transport domain in graphene nanoscale devices at room temperature.

This study is inspired from the recent experiment on direct chemical conversion of certain portions of graphene sheet into uniform boron nitride stripes (as barriers) without disturbing the structural arrangement of the graphene template in the direction of scalable synthesis of single-layer thin 2D integrated circuits $[7,8]$. In addition, this was also inspired from doping of graphene with different species [9]. Hybrid structures containing a mixture of carbon and h-BN nanodomains were of great interest for device engineers to fabricate different devices [7,8]. In particular, Lijie et al [8] synthesized

*Author for correspondence (penchalaiah.palla@vit.ac.in)
2D single layers containing large $\mathrm{BN}$ regions in graphene by thermal catalysis-assisted chemical vapour deposition technique. Another important progress towards graphene device fabrication is doping graphene using substitutional acceptors (boron) or donors (nitrogen) [9], hydrogen silsesquoxane films on graphene [10], polyethylene imine and diazonium salts [11], or other different techniques reviewed in ref. [12]. Owing to fast development in nanotechnology, we can expect that these types of nanostructures can be well fabricated on the atomic scale very soon. Therefore, the experiments stated above are specifically important for device applications of graphene, as they furnish a new path to engineer and induce the bandgap in graphene [13]. These experiments recently triggered studies on the electronic and transport properties of various h-BN and graphene heterostructures [14-19].

Recently, various types of resonant tunnelling diodes (RTDs) based on graphene have been proposed and explored [20,21]. One such is the graphene nanoribbon (GNR) based RTD. The operation of this type of devices is strongly dependent on the edge effects and the device shape [20]. The other types of RTD studied are based on graphene, which is epitaxially grown on $\mathrm{SiC}$ [21], which has a limitation of substrate-induced mobility degradation.

In this article, we propose a novel RTD based on graphene-h-BN heterostructures, where the two barriers are arranged by h-BN domains with a wide bandgap, as shown in figure 1. Graphene-based materials are reported on BN substrate in different stacking arrangements. Although the Bernal-stacked graphene on BN substrate induces some bandgap in graphene [22-24], which is useful for device applications, we will show later that the substrate-induced energy bandgap in graphene is not mandatory for doublebarrier resonant tunnelling diode (DBRTD) to operate properly. 




Figure 1. Simulated graphene RTD with two h-BN barriers. Here the graphene layer is supported on h-BN substrate. The dotted rectangle shows the magnified atomic structure of graphene contact/h-BN barrier/graphene quantum well.

\section{Materials and methodology}

ATLAS device simulator of SILVACO TCAD [25] is used for numerical calculation. Conceptually the DBRTD model is divided into three regions: the emitter region, central double barrier region and collector region. The emitter and collector regions are treated as in quasi-equilibrium, while the central region is considered as in non-equilibrium. Injection into the central region occurs from emitter quasi-bound states. Ballistic transport is assumed along $Y$-direction and no transport is allowed along $X$-axis. Also no inelastic phonon scattering is assumed. A single band effective mass is considered for calculation. The valence and conduction bands are treated parabolic and isotropic in the perpendicular plane. The current and carrier densities are integrated analytically over the k-space. Non-equilibrium carrier distribution in the RTD is consistently computed with the width, energy and occupancy of its scattering states using non-equilibrium Green's function (NEGF) formalism. The NEGF equations solved self-consistently with Poisson equation within an effective mass Hamiltonian model. The 2D device an effective-mass Hamiltonian $H_{0}$ [25] is given as follows:

$$
H_{0}=-\frac{h^{2}}{2}\left[\frac{\partial}{\partial x}\left(\frac{1}{m_{x}^{v}(x, y)} \frac{\partial}{\partial x}\right)+\frac{\partial}{\partial y}\left(\frac{1}{m_{y}^{v}(x, y)} \frac{\partial}{\partial y}\right)\right],
$$

where $m_{x, y}^{v}=$ effective mass in $x, y$ direction in valley $v$, $h=$ Plank's constant.

A nonlinear-Schrödinger equation also known as Quantum Transmitting Boundary Method (QTBM) is first solved in each 1D slice of the RTD to determine wavefunctions and Eigen energies and then for the creation of the energy grid $[26,27]$. In QTBM, the end contacts open boundary conditions are taken into the Hamiltonian, hence making the Eigen problem nonlinear and non-Hermitian. The resonant peak position and width are important in RTDs. These are predicted with the help of real and imaginary parts of the Eigen 
Table 1. Material parameters of graphene and h-BN.

\begin{tabular}{|c|c|c|c|}
\hline Materials & Graphene & h-BN & $\begin{array}{l}\text { Ref (graphene, } \\
\text { h-BN) }\end{array}$ \\
\hline Energy gap (eV) & 0 & 3.9 & {$[19,33]$} \\
\hline Electron affinity (eV) & 4.24 & 1.11 & [19] \\
\hline Permittivity & 4.22 & 4.2 & {$[34,35]$} \\
\hline $\begin{array}{l}\text { Effective mass of } \\
\text { electrons }\left(m_{\mathrm{e}}\right)\end{array}$ & 0 & 0.26 & {$[36,37]$} \\
\hline Effective mass of holes $\left(m_{\mathrm{h}}\right)$ & 0 & 0.47 & {$[36,37]$} \\
\hline $\begin{array}{l}\text { Effective density of states } \\
\text { in conduction band }\left(\mathrm{cm}^{-3}\right)\end{array}$ & $10^{13}$ & $2.1 \times 10^{19}$ & {$[37,38]$} \\
\hline $\begin{array}{l}\text { Effective density of states } \\
\text { in valence bond }\left(\mathrm{cm}^{-3}\right)\end{array}$ & $10^{13}$ & $2.1 \times 10^{19}$ & {$[37,38]$} \\
\hline
\end{tabular}

energies. These Eigen energies also help us to place enough number of grid points in critical regions.

Then, equations (2) and (3), the quantum transport (NEGF) equations, of carriers is solved.

$$
\begin{aligned}
& G^{R}=\left(E-H_{0}-\sum_{E}^{R}-\sum_{C}^{R}\right)^{-1}, \\
& \left(E-H_{0}-\sum_{E}^{R}-\sum_{C}^{R}\right) G^{<}=\left(\sum_{E}^{<}+\sum_{C}^{<}\right) G^{A} .
\end{aligned}
$$

Here, $\Sigma_{E, C}^{R}$ are retarded self-energies of emitter and collector semi-infinite contacts. $E$ is an electron (or hole) energy. $\Sigma_{E, C}^{<}$ are less-than self-energies of contacts. The solution of the above equations are a retarded Green's function $G^{R}(E)$ and a less-than Green's function $G^{<}(E)$, whose diagonal elements have a meaning of local density of states and carrier density as a function of energy, respectively. Here $G^{A}(E)$ is the advanced Green's function and $G^{A}(E)=\left(G^{R}(E)\right)^{\dagger}$, $\dagger$ expression indicates complex conjugate of transpose of matrix $\left(G^{R}(E)\right)$.

Equations (2) and (3) are solved on a non-uniform energy grid. Carrier density and current density are computed by integrating Green's function on 2D transverse k-space analytically. The various parameters in $\mathrm{m}$-th sub-band are estimated as follows [25]:

Transmission coefficient:

$$
T_{m}=\operatorname{tr}\left\{G^{R} \Gamma_{L} G^{A} \Gamma_{R}\right\}, \Gamma_{L, R}=2 \Im m\left\{\Sigma_{E, C}^{R}\right\},
$$

where $\Gamma_{L, R}$ indicates (matrix) energy broadening in $\mathrm{eV}$ for left and right electrodes, respectively.

Density of states:

$$
\operatorname{DOS}_{m}\left(E, x_{i}\right)=2 \Im m\left\{G_{m m i i}^{R}(E)\right\} .
$$

Electron density per energy:

$$
N_{m}\left(E, x_{i}\right)=\Im m\left\{G_{m m i i}^{<}(E)\right\} .
$$

Current density per energy:

$$
J_{m}\left(E, x_{i}\right)=\frac{2 e}{h} \Re e\left\{h_{m m i+1 i} G_{m m i i+1}^{<}(E)\right\} .
$$

The total current density through the 2D device is computed by integrating current densities over all 1D slices.

In this article we are reporting DBRTD using graphene and h-BN heterostructures on h-BN substrate. Here, graphene is used for emitter, collector and well, and h-BN domains are used for tunnel barriers. The important material parameters used for simulation are extracted from various experiments and $a b$ initio calculations published recently on h-BN and graphene are listed in table 1.

\section{Results and discussion}

The results of both $\mathrm{n}$ - and p-type DBRTDs are discussed in the following subsections.

\subsection{Graphene/h-BN RTD with n-type doped access regions}

The simulated DBRTD is having emitter and collector regions as access regions with $20 \mathrm{~nm}$ long. The emitter and collector regions are having doping of $5 \times 10^{12} \mathrm{~cm}^{-2}$ with n-type dopant. Doped graphene can be realized using the methods specified in refs [9-12]. The barriers have dimensions of $1.3 \mathrm{~nm}$ each with no doping. The central well has a width of $4.3 \mathrm{~nm}$ with intrinsic doping.

At $0.5 \mathrm{~V}$ applied bias, the edge (or potential) profile of conduction band of DBRTD is shown in figure 2. The Eigen energies related to the lowest Eigen states are shown as horizontal lines for emitter (black) and quantum well region (red) respectively. At small applied biases, the energy of well state (red) (i.e., resonant energy) is at higher level than the energy of emitter states.

The resonant state (red-wave function 2) corresponding to resonant energy (as displayed in figure 2), which is in between double barrier structure (i.e., in well region), is precisely localized with almost no leakage, as shown in figure 3 . This figure, actually represents the square of absolute value of quasi-bound state for emitter (wavefunction 1) and well regions (wavefunction 2), respectively. The emitter quasibound state (black, wavefunction 1) indicates significant leakage into the emitter region even though it is localized in front of the emitter side barrier.

The resonant energy is the Eigen energy at which electrons tunnel through the barrier-well-barrier structure. At resonant energy, whose value is $0.01 \mathrm{eV}$, there is a sharp peak on the spectra of current density and transmission probability as shown in figure 4. It is evident from the results displayed in figure 2 (horizontal line with red-open circle) that a low-energy transmission peak (resonance) exists at energy $0.01 \mathrm{eV}$. The applied positive bias (or its corresponding electric field) is responsible to pull down the potential well (or quantum well) below zero potential energy and correspondingly resonant energy shifted close to 0.01 $\mathrm{eV}$. At incident energy $0.01 \mathrm{eV}$, there is a unity peak in transmission coefficient ( $Y$-axis of figure 4 is shown in $\log$ scale) that comes out above the smoothly varying electron transmission background. The reduction in transmission 
coefficient beyond $0.01 \mathrm{eV}$ is due to reduction (towards zero) of velocity of an incident electron from the emitter (left side) in the region before the first h-BN potential barrier. This causes large velocity mismatch, which in turn gives rise to low transmission and large reflection. However, the slight increase in overall transmission at high energy is due to increase in background transmission. Since current is proportional to transmission coefficient, the sharp (resonant) peak in current density occurs exactly at same energy as transmission coefficient. Hence, there is a sharp peak in current density at $0.01 \mathrm{eV}$. Therefore, the current density per energy beyond $0.01 \mathrm{eV}$ tends to zero, as shown in figure 4 . The total current will be the integration of overall current density spectrum at different energies, out of which the main element of contribution is from the resonant peak observed at $0.01 \mathrm{eV}$.

In figure 5, the sharp resonant peak (green) is due to strong confinement of well-defined bound states, while the spread of emitter DOS (red) is the indication of leakage of quasi-bound states into emitter region. By integrating local DOS over the

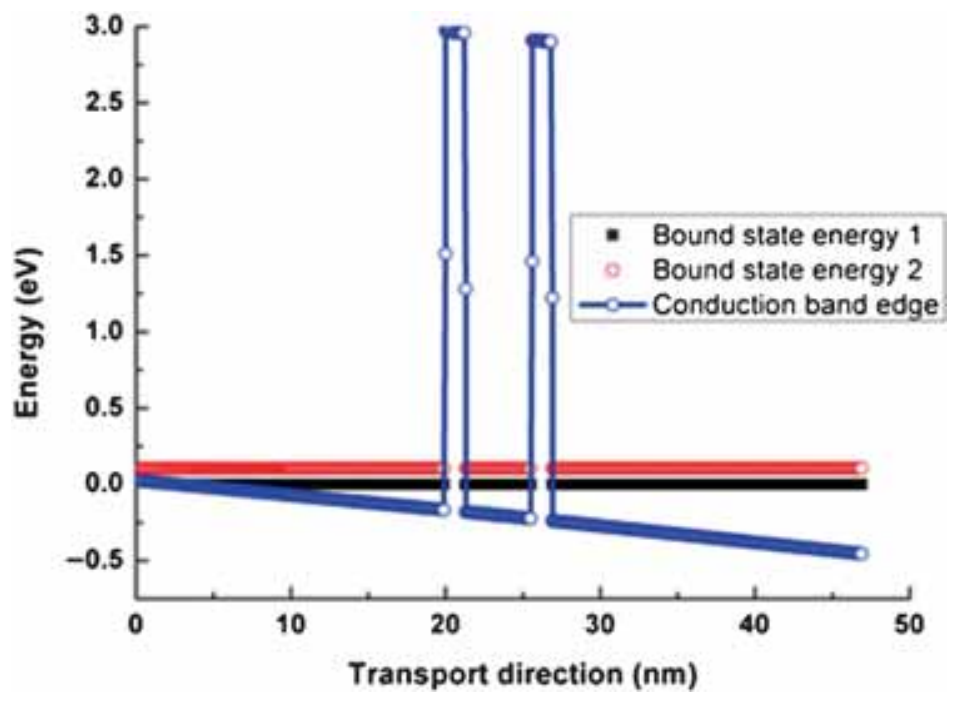

Figure 2. The Eigen energies in the emitter (horizontal line with black solid square) and in the well (horizontal line with red open circle) together with edge profile of conduction band (blue line and open circle) of DBRTD along the transport direction is shown at $0.5 \mathrm{~V}$ applied bias.

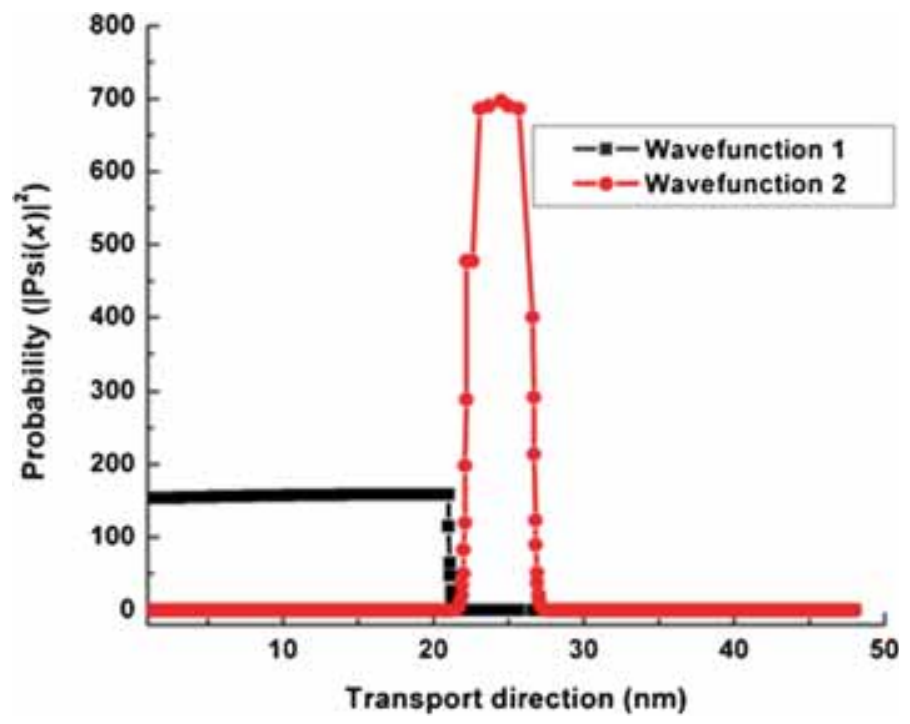

Figure 3. Localized resonant state (red line with solid circle) and emitter-quasi bound state (black line with solid square) in well region and emitter region, respectively, at $V_{\text {bias }}=0.5 \mathrm{~V}$. 
energy range, the total carrier density will be obtained. The proper resolution of the resonant peaks in the local DOS as a function of energy will in turn help to treat electrostatics properly.

In addition, as shown in figure 6 , it is observed that when the bias voltage is increased from 0 to $0.5 \mathrm{~V}$ the electron density in quantum well increases and further increase in bias voltage from 0.5 to $0.54 \mathrm{~V}$ resulted in the reduction of electron density, which is attributed to the resonant tunnelling. The electrical behaviour of DBRTD is explored in terms of transmission spectrum, DOS and electron density in quantum well as shown in figures 4, 5 and 6, respectively. Figure 7 (black) shows an increase in total current density due to background transmission and resonance in quantum

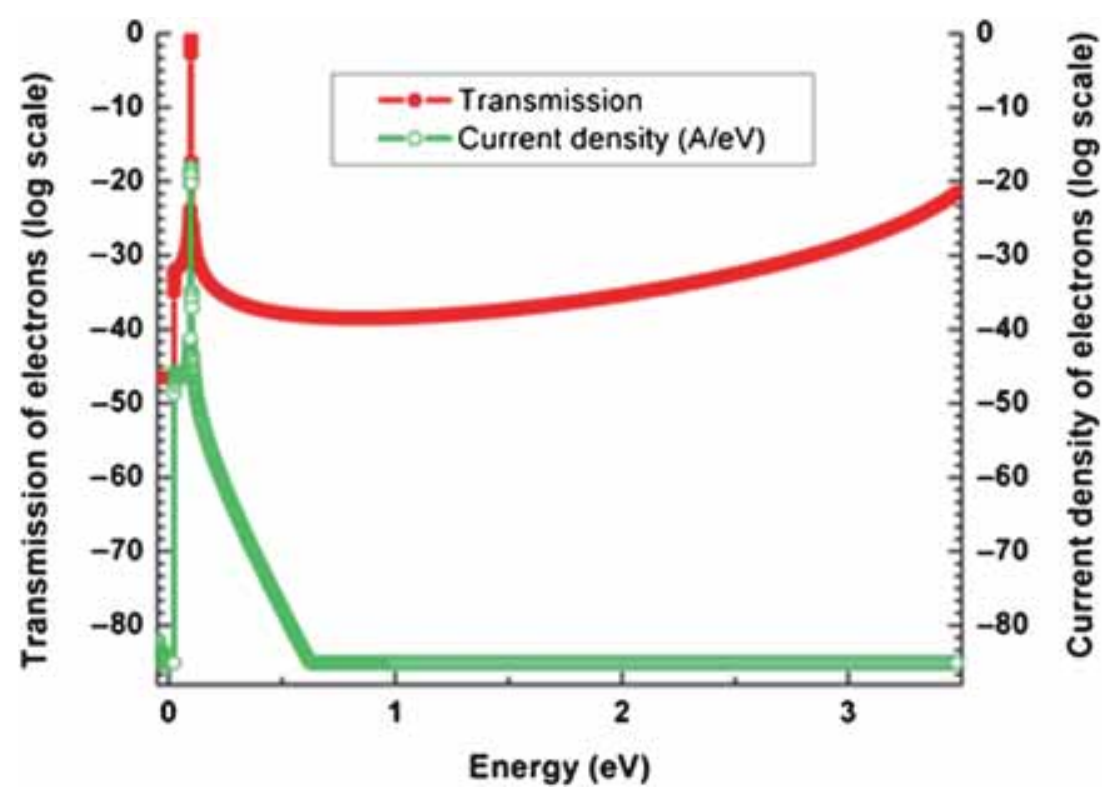

Figure 4. Current density (green line with open circle) and transmission (red line with solid circle) spectra at applied bias of $0.5 \mathrm{~V}$.

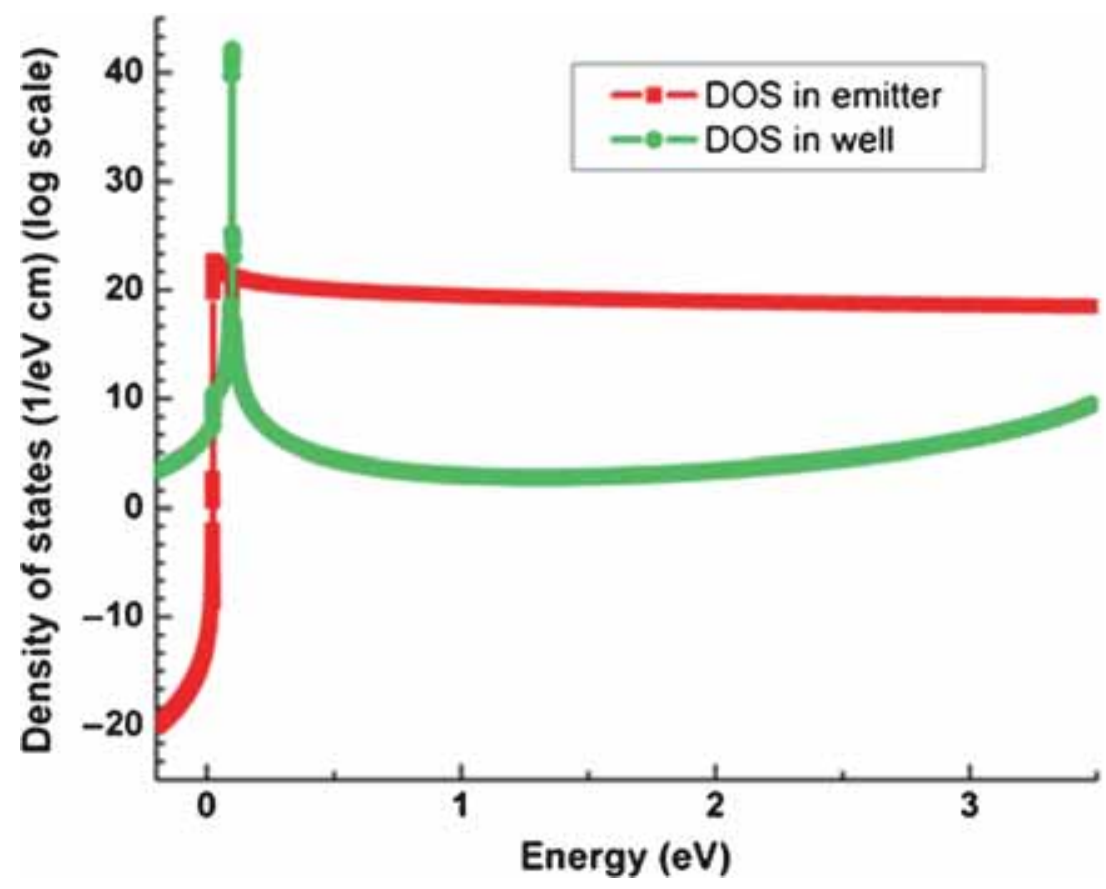

Figure 5. Local density of states (DOS) as a function of energy in the well (green line with solid circle) and emitter (red line with solid square) regions. 


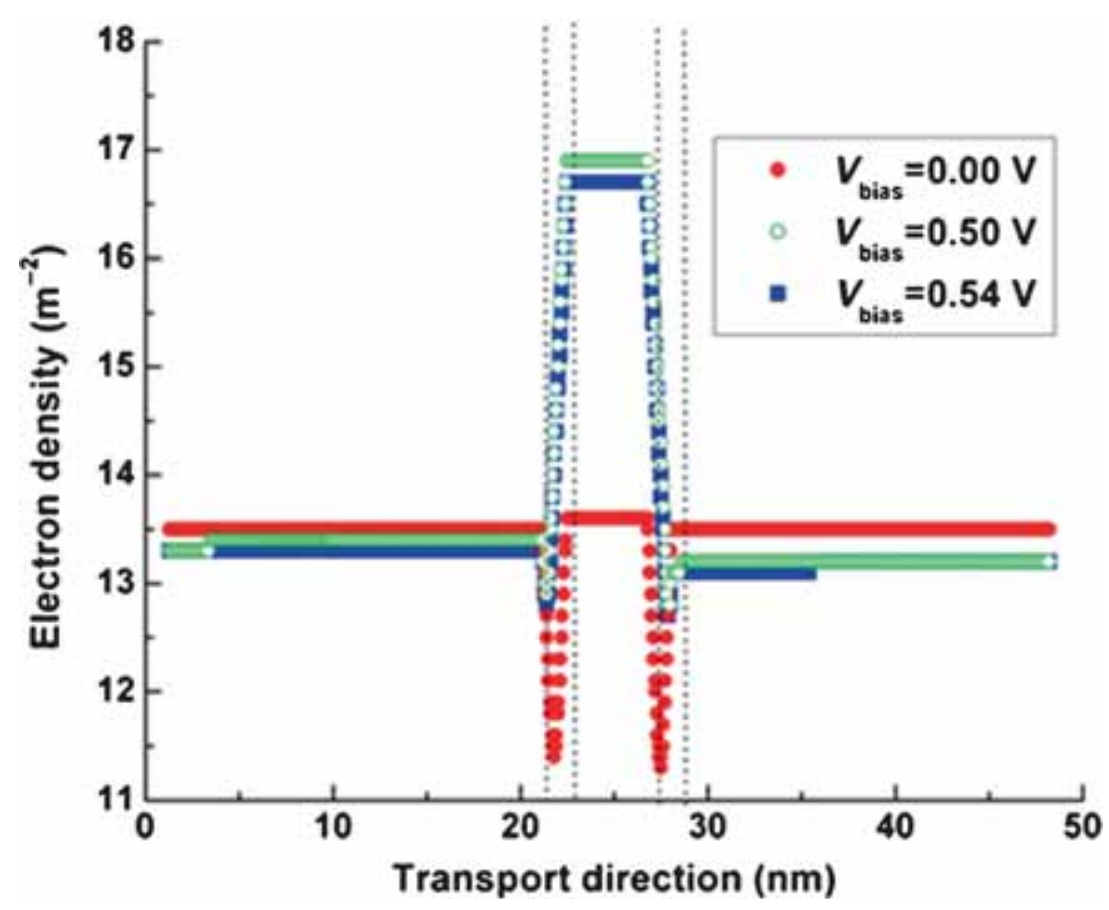

Figure 6. Electron density profile at biases of $0,0.5$ and $0.54 \mathrm{~V}$.

well, as explained in figures 4 and 6 from 0 to $0.5 \mathrm{~V}$. A sudden decrease in total current density at $0.54 \mathrm{~V}$ (at valley point) is due to non-availability of states in emitter region just in front of the first barrier as explained below: Figure 2 highlights the large h-BN barriers (approximately $3.0 \mathrm{eV}$ ). This gives rise to the quantization of carrier states in the quantum well (figure 3 (red)), which generates strong resonant peak in the transmission (figure 4) and DOS (figure 5) at $0.01 \mathrm{eV}$, respectively. The outcome of the strong resonant tunnelling, figure 7 , shows a strong negative differential resistance (NDR) for pristine graphene $\left(E_{\mathrm{G}}=0 \mathrm{meV}\right)$. When finite energy gap of $100 \mathrm{meV}$ is induced in graphene through h-BN substrate, peak to valley current ratio (PVR) increased even though both peak and valley currents are reduced, as shown in figure 7 (red line with triangle).

In conventional RTDs, the resonant current at finite bias is zero because of the occurrence of resonant level in front of the bandgap of the emitter region. This situation is not valid for the case of pristine graphene. The shown NDR behaviour is interpreted as below. In spite of zero bandgap of graphene at Dirac point corresponding to $k_{y} \equiv K_{y}=1.209 a_{\mathrm{cc}}$, a finite energy gap $\hat{\mathrm{E}}\left(k_{y}\right)=2 t \mid 1-2 \cos \left(0.866 a_{\mathrm{cc}} k_{y}\right)$ still appears for other momenta $k_{y} \neq K_{y}$ [28], where $t=2.5 \mathrm{eV}$ and $a_{\mathrm{cc}}$ is the carbon to carbon bond length.

For example, for $k_{y}=K_{y}+0.025 / a_{\mathrm{cc}} \sqrt{3}, \hat{\mathrm{E}}_{\mathrm{g}}\left(k_{y}\right)$ is approximately $0.12 \mathrm{eV}$, which is the energy gap responsible for switching-off resonant current at valley point at high bias. The valley region of the $I-V$ characteristic is narrower than conventional RTDs, this is due to band to band tunnelling through small bandgap of graphene, as explained above [29]. In graphene when finite bandgap is introduced
[22-24,30,31], it is found that peak-to-valley current ratio (PVR) increases; while there is reduction in both the valley and the peak currents. The PVR reaches approximately 3 for $E_{\mathrm{G}}=0$ and 6.4 for $E_{\mathrm{G}}=100 \mathrm{meV}$, respectively. Our study shows that the PVR values obtained are higher than the reported best PVR values for conventional semiconductor devices [10] as well as graphene RTDs [20,21].

Figure 8 illustrates the $I-V$ characteristics by taking into consideration the effect of quantum well width QW on the RTD operation. The results indicate that with increase in the well width QW the current valleys shifts towards the low bias and slowly weakens the NDR effect. The possible reason for this particular effect is due to the influence of number of energy levels in the quantum well and the available spacing between them. The complex $I-V$ curve with a low PVR is the result of multi-level contribution due to large QW (5.1 nm).

\subsection{Graphene/h-BN RTD with p-type doped access regions}

In this section, we focus on graphene DBRTD using hBN barriers with p-type doping. The width and separation distance of h-BN used are 1.3 and $5.1 \mathrm{~nm}$, respectively. The $20 \mathrm{~nm}$ wide emitter and collector are doped with p-type with a concentration of $5 \times 10^{12} \mathrm{~cm}^{-2}$. For holes a single band model is considered. The hole effective masses were taken as $m_{\mathrm{h}}$ (graphene $)=0$, and $m_{\mathrm{h}}(\mathrm{hBN})=0.47$. The other relevant parameters used in the simulation are mentioned in table 1.

Figure 9 shows valence band profile at $-0.12 \mathrm{~V}$. Compared with other Eigen energy, the resonant energy in the well (solid red circle) is much higher than the energy of the 


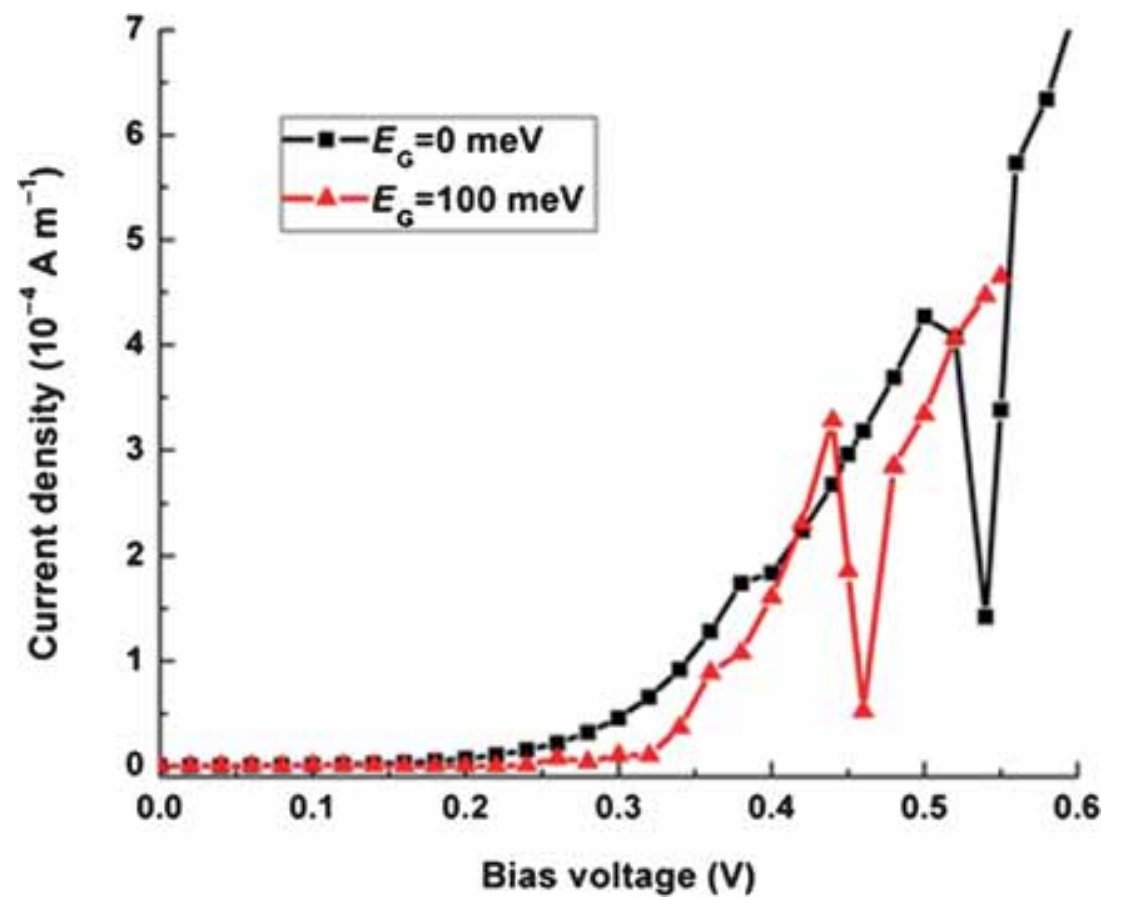

Figure 7. $I-V$ characteristics of DBRTD with n-type doping for energy gaps $E_{\mathrm{G}}=0$ and $100 \mathrm{meV}$.

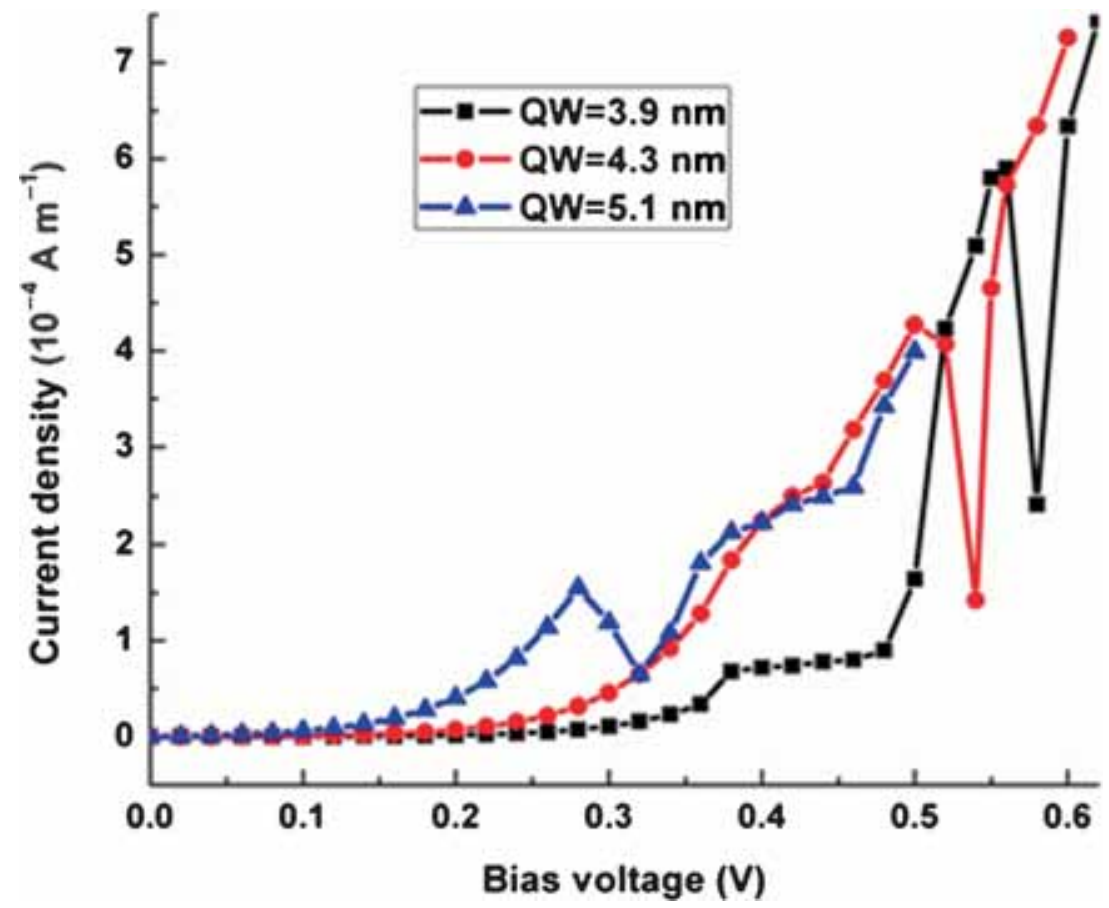

Figure 8. $I-V$ characteristics for different well widths (QW).

other state at low voltage. Figure 10 shows the corresponding probability functions of resonant states, which clearly indicates negligible leakage of states outside of the barrier due to high localization (red) in the well. For other quasi-bound states although the nature of the localized state is same, the magnitude is significantly small.
Figure 11 shows a sharp narrow peak for hole current density and transmission spectra at the resonant energy. It is understood from the results displayed in figure 9 (horizontal line with red solid circle) that a low-energy transmission peak (resonance) exists at energy $-0.023 \mathrm{eV}$. The applied negative bias is responsible to pull up the quantum well above 


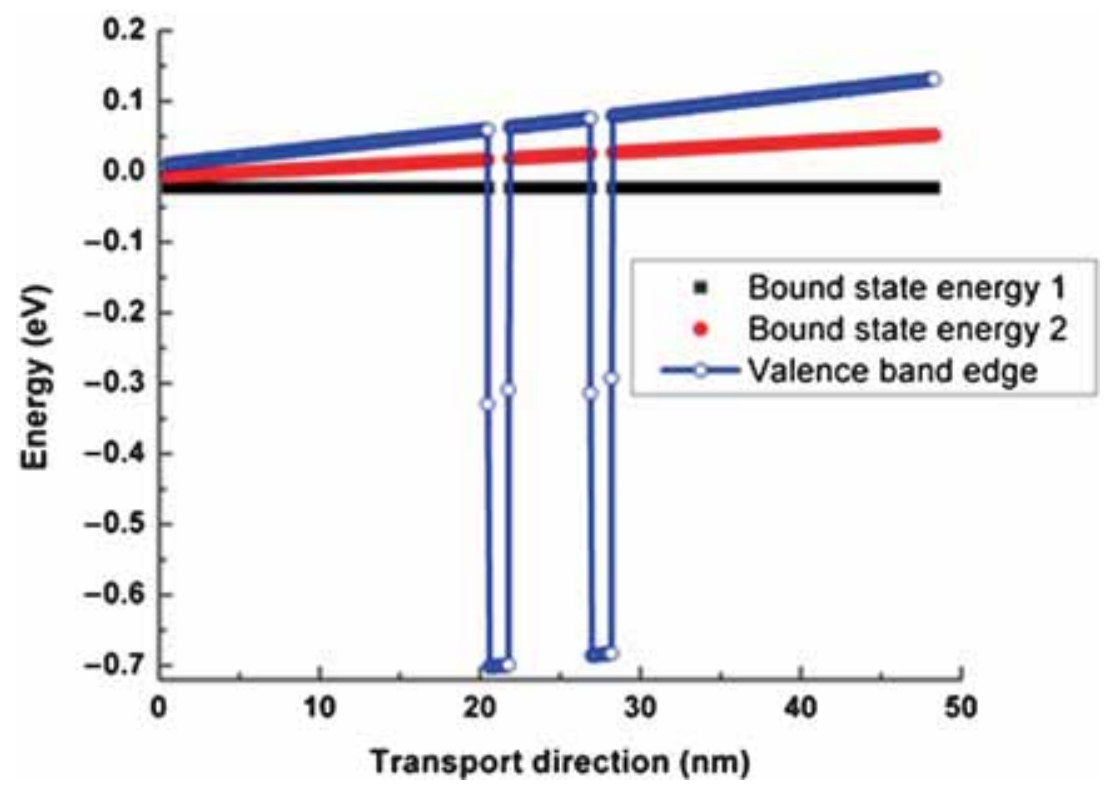

Figure 9. The Eigen energies in the emitter (horizontal line with black solid square) and in the well (horizontal line with red solid circle) together with edge profile of valence band (blue line with open circle) of DBRTD along the transport direction is shown at $-0.12 \mathrm{~V}$ applied bias.

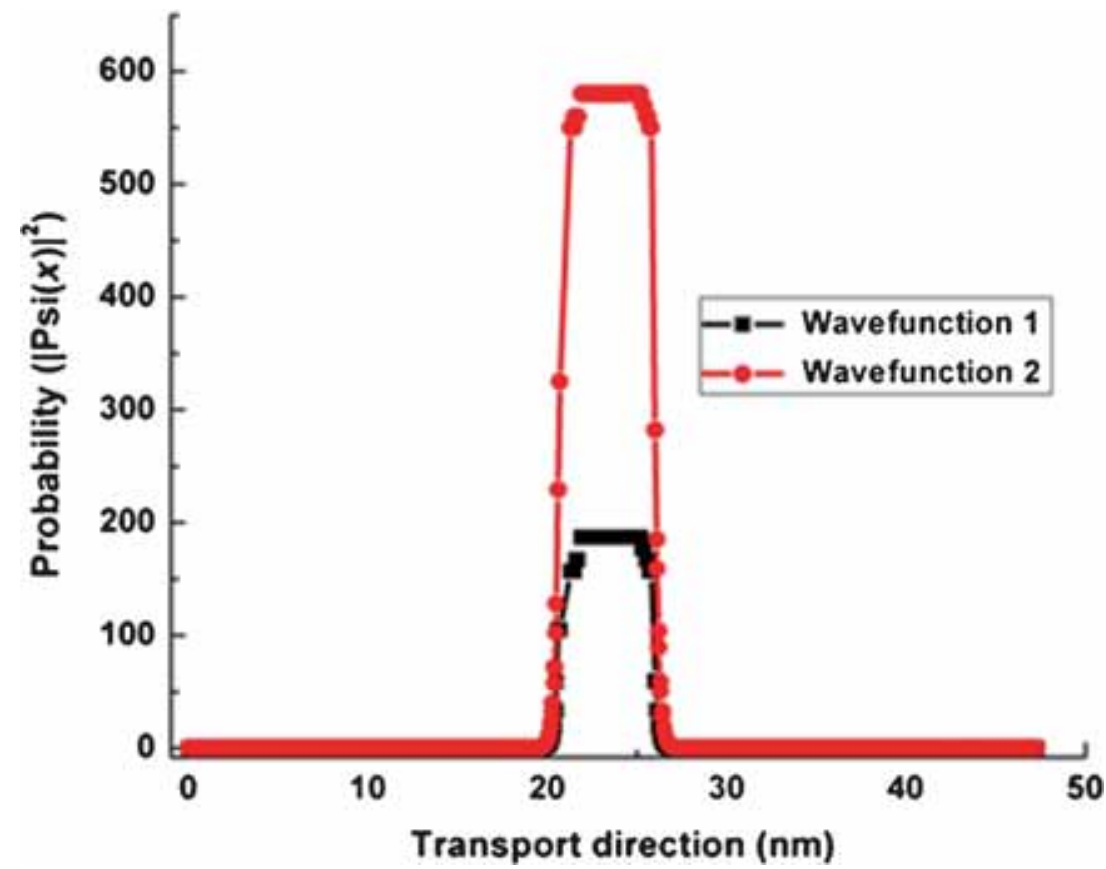

Figure 10. Wavefunctions corresponding to Eigen energies shown in figure 9.

zero potential energy and correspondingly resonant energy shifted close to $-0.023 \mathrm{eV}$. At incident energy $-0.023 \mathrm{eV}$, there is a unity peak in hole transmission coefficient $(Y$-axis of figure 11 is shown in log scale) that comes out below the smoothly varying hole transmission background similar to electrons in figure 4 . The reduction in transmission coefficient beyond $-0.023 \mathrm{eV}$ is due to reduction (towards zero) of velocity of an incident hole from the emitter (left side) in the region before the first $\mathrm{h}$-BN potential barrier. This causes large velocity mismatch, which in turn gives rise to low transmission and large reflection. However, the slight increase in overall transmission at high energy is due to increase in the background hole transmission. Since current is proportional to transmission coefficient, the sharp (resonant) 
peak in current density occurs exactly at same energy as the transmission coefficient. Hence, there is a sharp peak in current density at $-0.023 \mathrm{eV}$. Therefore, the current density per energy beyond $-0.023 \mathrm{eV}$ tends to be zero, as shown in figure 9.

In addition, as shown in figure 12 for $\mathrm{QW}=5.1 \mathrm{~nm}$, when the bias voltage is increased from 0 to $-0.12 \mathrm{~V}$ the hole density in quantum well increases and further increase in bias voltage from -0.12 to $-0.13 \mathrm{~V}$ resulted in the reduction in hole density, which is attributed to the hole resonant tunnelling similar to electron resonant tunnelling (figure 7). An increase in total current density (figure 12) from 0 to $-0.12 \mathrm{~V}$ is due to background transmission and resonance in quantum well. A sudden decrease in total current density at $-0.13 \mathrm{~V}$ (at valley point) is due to the non-availability of states in emitter region just in front of first barrier. The

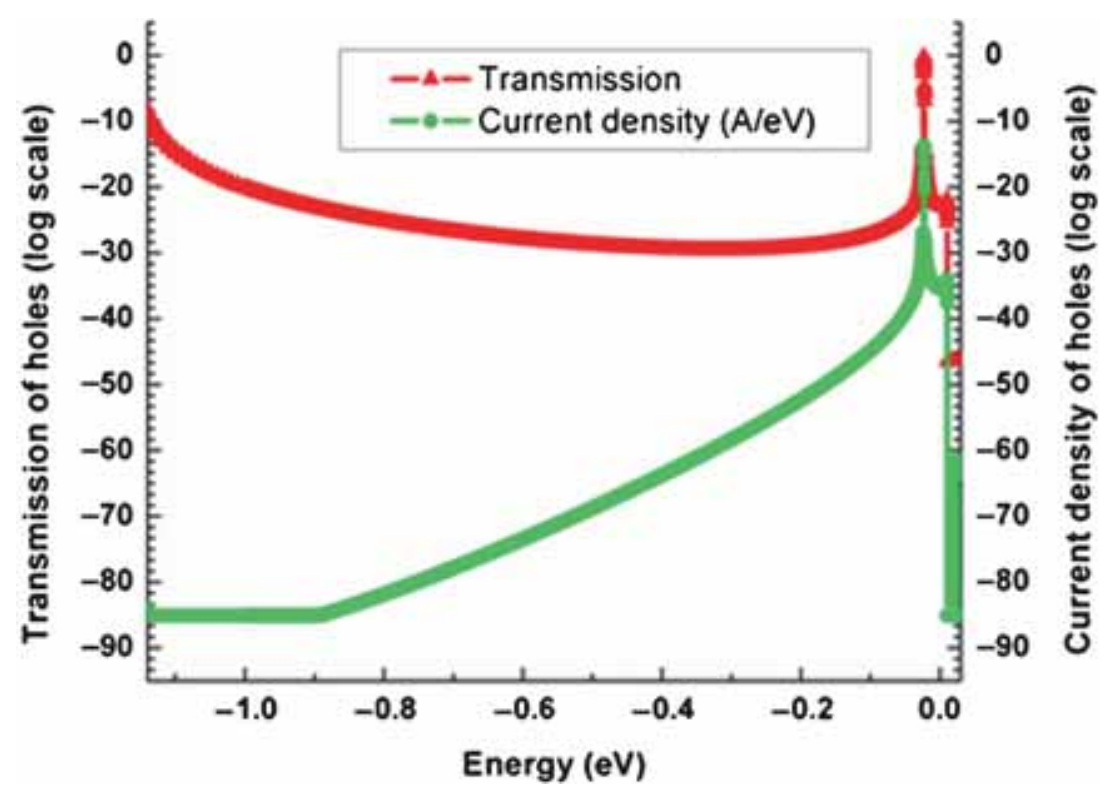

Figure 11. Current density (green line with solid circle) and transmission (red line with triangle) spectra observed for $-0.12 \mathrm{~V}$ bias voltage.



Figure 12. $I-V$ characteristics for different well widths $(\mathrm{QW})$. 
outcome of the strong resonance and as a consequence of the tight confinement of hole resonant states in the quantum well, figure 12 shows a pronounced NDR behaviour in electrical characteristics of p-type device even for pristine graphene $\left(E_{\mathrm{G}}=0 \mathrm{meV}\right)$. Here, we observed a PVR of 3.40, 7.06 and 7.83 for well widths $3.9,4.3$ and $5.1 \mathrm{~nm}$, respectively.

We observed that, in addition to the increase in the width of the barrier due to the large bandgap of h-BN, there is a strong reduction in the total device current. Therefore, a barrier thickness of less than $2 \mathrm{~nm}$ is desirable to obtain the proper resonant effects.

\subsection{Effect of non-ideal behaviour of $h-B N$ edges}

All the results demonstrated so far are for RTDs with ideal edge behaviour between h-BN and graphene regions. It is an experimentally challenging task to maintain precise edge between h-BN and graphene interface as there is possibility of edge-related disorder, which altogether can affect transport parameters. In our device the rough edges between $\mathrm{h}-\mathrm{BN}$ and graphene are perpendicular to the transport direction, which is not the case for disorder GNRs [32] where the direction of the rough edges is parallel. Owing to the existence of the edge disorder of $\mathrm{h}-\mathrm{BN}$ along the OX direction makes the width of graphene quantum well and barrier of $h$ $\mathrm{BN}$ non-uniform. This edge disorder may change the position of bound levels in the quantum well and also affect the device geometry.

\section{Conclusions}

In summary, a graphene RTD with h-BN as double barrier material supported on h-BN substrate is explored by means of Poisson and NEGF equations within the effective mass Hamiltonian model. The resonant tunnelling and the resulting NDR behaviour for both n- and p-type RTDs were observed. The effect of device parameters such as the barrier width and the quantum well width on the $I-V$ characteristics was analysed. The PVR of approximately 3 for gapless and higher than 7 for finite gap (due to Bernal stacking of graphene on h-BN) are achieved at room temperature for n-type RTD. These results suggest that the bandgap engineering of h-BN and graphene opens a new way for high-performance electronic devices for post-silicon era with the following predictions: (1) The smaller lengths of quantum wells of graphene/h-BN DBRTDs enable the prospect of very-fast transit times of tunnelling carriers compared to its conventional counter parts with tens of nanometres wide. This feature, combined with NDR form of device characteristics, has potential for applications in high-frequency oscillators. (2) The p-type DBRTDs will show large resonant tunnelling and higher PVR compared to n-type DBRTDs for the same device dimensions, which is due to smaller h-BN barrier heights of p-type devices than n-type devices. Moreover, the p-type devices can be operated at low bias conditions than n-type devices. (3) Although the Bernal-stacked graphene on BN substrate induces some bandgap in graphene, which is useful for device applications, the substrate-induced energy bandgap in graphene is not mandatory for DBRTD to operate properly. (4) Owing to large bandgap of h-BN, a barrier thickness of less than $1.5 \mathrm{~nm}$ is desirable to obtain the proper resonant effects. Finally, the graphene and h-BN material parameters used in this study will be helpful for commercial device simulators to design new nanoscale devices.

\section{References}

[1] Li S, Lijie C, Hao L, Pavel B S, Chuanhong J, Jie N et al 2010 Nano Lett. 103209

[2] Ponomarenko L A, Yang R, Mohiuddin T M, Katsnelson M I, Novoselov K S, Morozov S V et al 2009 Phys. Rev. Lett. 102 206603

[3] Zomer P J, Dash S P, Tombros N and Van Wees B J 2011 Appl. Phys. Lett. 99232104

[4] Dean C R, Young A F, Meric I, Lee C, Wang L, Sorgenfrei S et al 2010 Nat. Nanotechnol. 5722

[5] Jiamin X, Javier S-Y, Danny B, Philippe J, Aparna D, Watanabe K et al 2011 Nat. Mater. 10282

[6] Régis D, Yang W, Victor W B, William R, Hsin-Zon T, Qiong W et al 2011 Nano Lett. 112291

[7] Yongji G, Gang S, Zhuhua Z, Wu Z, Jeil J, Weilu G et al 2014 Nat. Commun. 54193

[8] Lijie C, Li S, Chuanhong J, Deep J, Dangxin W, Yongjie L et al 2010 Nat. Mater. 9430

[9] Panchakarla L S, Subrahmanyam K S, Saha S K, Govindaraj A, Krishnamurthy H R, Waghmare U V et al 2009 Adv. Mater. 214726

[10] Brenner K and Raghunath M 2010 Appl. Phys. Lett. 96 063104

[11] Damon B F, Roksana G-M, Vasili P, Yu-Ming L, George S T, James C T et al 2008 Nano Lett. 9388

[12] Hongtao L, Yunqi L and Daoben Z 2011 J. Mater. Chem. 21 3335

[13] Angel R 2010 Nat. Mater. 9379

[14] Jun H, Ke-Qiu C, Zhi-Qiang F, Li-Ming T and Hu W P 2010 Appl. Phys. Lett. 97193305

[15] Xu B, Lu Y H, Feng Y P and Lin J Y 2010 J. Appl. Phys. 108 073711

[16] Basheer E A, Prakash P and Swapan K P 2011 New J. Phys. 13053008

[17] Junwen L and Vivek B S 2011 Appl. Phys. Lett. 98013105

[18] Gyungseon S and Jing G 2011 Appl. Phys. Lett. 98143107

[19] Gianluca F, Alessandro B, Samantha B and Giuseppe I 2012 ACS Nano 62642

[20] Hansen T, Kai-Tak L, Sharjeel B K and Gengchiau L 2009 J. Appl. Phys. 105084317

[21] Nguyen V H, Bournel A and Dollfus P 2011 Semicond. Sci. 26125012

[22] Gianluca G, Petr A K, Geert B, Paul J K and Jeroen van den B 2007 Phys. Rev. B 76073103

[23] Neerav K and Saroj K N 2011 Nano Lett. 115274 
[24] Penchalaiah P and Raina J P 2012 Innovative systems design and engineering 327

[25] ATLAS user's manual 2013 (Santa Clara, CA: Silvaco Inc.)

[26] Chenjing L F and William R F 1994 J. Appl. Phys. 762881

[27] Gerhard K, Roger K L, Chris B R, Chenjing L F and William R F 1998 VLSI Design 6107

[28] Castro Neto A H, Guinea F, Peres N M R, Novoselov K S and Geim A K 2009 Rev. Mod. Phys. 81109

[29] Nam Do V, Dollfus P and Lien Nguyen V 2006 J. Appl. Phys. 100093705

[30] Yang X, Zhendong G, Huabin C, You Y, Jiechao L et al 2011 Appl. Phys. Lett. 99133109

[31] Yingcai F, Mingwen Z, Zhenhai W, Xuejuan Z and Hongyu Z 2011 Appl. Phys. Lett. 98083103
[32] Mucciolo E R, Castro Neto A H and Lewenkopf C H 2009 Phys. Rev. B 79075407

[33] Nguyen V H, Mazzamuto F, Bournel A and Dollfus $2012 \mathrm{~J}$. Phys. D: Appl. Phys. 45325104

[34] Choongyu H, David A S, Sung-Kwan M, William R, Ariel I, Yuegang Z et al 2012 Sci. Rep. 2590

[35] Gang S, Yuranan H, Zheng L, Yongji G, Weilu G, Bo L et al 2014 Nano Lett. 141739

[36] AlZahrani A Z and Srivastava G P 2009 Braz. J. Phys. 39694

[37] Michael E L, Sergey L R and Michael S S 2001 Properties of advanced semiconductor materials: GaN, AlN, InN, BN, SiC, $\mathrm{SiGe}$ (New York, Wiley) ISBN: 978-0-471-35827-5

[38] Novoselov K S, Geim A K, Morozov S V, Jiang D, Zhang Y, Dubonos S V et al 2004 Science 306666 